Article

\title{
An Efficient Synthesis of Aryl-Substituted Pyrroles by the Suzuki-Miyaura Coupling Reaction of SEM-Protected Pyrroles
}

\author{
Keli Cui ${ }^{1}$, Meng Gao ${ }^{2}$, Hongyi Zhao ${ }^{2}$, Dongfeng Zhang ${ }^{2, *} \mathbb{D}$, Hong Yan ${ }^{1, *}$ \\ and Haihong Huang ${ }^{2, *}$ \\ 1 College of Life Science and Bio-engineering, Beijing University of Technology, 100 Ping Le Yuan, \\ Chaoyang District, Beijing 100124, China; ck10615@163.com \\ 2 State Key Laboratory of Bioactive Substances and Function of Natural Medicine, Beijing Key Laboratory of \\ Active Substance Discovery and Druggability Evaluation, Institute of Materia Medica, Peking Union \\ Medical College and Chinese Academy of Medical Sciences, 1 Xian Nong Tan Street, Beijing 100050, China; \\ gaomengss@imm.ac.cn (M.G.); zhaohongyicool@imm.ac.cn (H.Z.) \\ * Correspondence: zdf@imm.ac.cn (D.Z.); hongyan@bjut.edu.cn (H.Y.); joyce@imm.ac.cn (H.H.)
}

Received: 19 March 2019; Accepted: 20 April 2019; Published: 22 April 2019

check for updates

\begin{abstract}
An efficient arylation of SEM-protected pyrroles by the Suzuki-Miyaura coupling reaction has been developed. The reaction can be carried out under mild conditions to provide aryl-substituted pyrroles in moderate to excellent yields. The scope and limitations of the methodology were evaluated, and the reaction was tolerant of a wide range of functionalities. Compared to the reported methods, the protocol has some advantages, such as commercially available materials, no debrominated by-products being formed, and the amine-protecting group being stable under the reaction conditions. The synthetic utility of the product has also been demonstrated, with several common transformations of the aryl-substituted pyrrole product being conducted. This protocol will offer the opportunity to explore other metal-catalyzed cross-coupling reactions employing SEM-protected pyrroles.
\end{abstract}

Keywords: aryl-substituted pyrroles; Suzuki-Miyaura coupling reaction; SEM-protected pyrroles

\section{Introduction}

Aryl-substituted pyrroles are an important structural motif in both pharmaceuticals and natural products alike, and display a wide range of interesting biological activities [1]. Aryl pyrroles bearing an ester functional group are commonly used in organic synthesis and medicinal chemistry as synthetic intermediates [2-6]. There are a great deal of methods for the preparation of aryl-substituted pyrroles with this pendant ester group, and generally, the pyrrole core is formed from functionalized precursors with complex structures employing various catalysts and ligands [7-14]. As our aim was to construct a small library of aryl-substituted pyrroles for biological activity assays, we found that it was inefficient to build these required pyrrole structures from some non-commercial starting materials. If the aryl-substituted pyrroles could be synthesized from commercially available pyrrole compounds, the approach will be simple, straightforward, and efficient. The coupling reaction is a powerful method in the formation of $\mathrm{C}\left(\mathrm{sp}_{2}\right)-\mathrm{C}\left(\mathrm{sp}_{2}\right)$ bonds, and the aryl-substituted pyrroles could be synthesized by the Stille-Migita cross-coupling reaction [15,16], decarboxylative coupling reaction [16, 17], metal-catalyzed desulfitative coupling reaction [18], and Suzuki-Miyaura coupling reaction [16]. Considering the availability of materials and the operability of the reaction, the Suzuki-Miyaura coupling reaction may be the most suitable method to prepare aryl pyrroles from arylboronic acids and bromopyrroles. However, without protection of the nitrogen of the pyrrole moiety, debromination readily occurs, and the debrominated compounds are generally obtained in a large percentage $[19,20]$. 
A $t$-butyloxy carbonyl (BOC) group is the traditional choice for an amine-protecting group; however, it is well known that these groups are often unstable under general Suzuki-Miyaura coupling reaction conditions $[3,19,21]$. Therefore, the development of a high-throughput synthesis of aryl-substituted pyrroles with protection for subsequent application via a Suzuki-Miyaura coupling reaction is highly desirable. SEM (2-(trimethylsilyl)ethoxymethyl) represents a versatile and robust protecting group for nitrogen heterocycles that can readily be removed under a variety of deprotection conditions [22-27]. Herein, we present a method that allows for the efficient arylation of SEM-protected pyrroles via the Suzuki-Miyaura coupling reaction. This methodology provides the desired products in high yields for a wide range of substrates.

\section{Results and Discussion}

Initially, phenylboronic acid (1a) and 4-bromopyrrole (2a) were chosen as the model substrates for the Suzuki-Miyaura coupling reaction. In the presence of $\mathrm{Pd}\left(\mathrm{PPh}_{3}\right)_{4}\left(0.1\right.$ equiv.) and $\mathrm{Na}_{2} \mathrm{CO}_{3}$ (2 equiv.), the reaction between 1a and 2a in dioxane/ $\mathrm{H}_{2} \mathrm{O}(4: 1)$ at $90{ }^{\circ} \mathrm{C}$ afforded the desired coupling product (3a) in 61\% yield (Table 1, entry 1). However, the use of $\mathrm{Pd}^{(\mathrm{II})}$ salts, such as $\mathrm{Pd}\left(\mathrm{PPh}_{3}\right)_{2} \mathrm{Cl}_{2}$, $\mathrm{Pd}(\mathrm{AcO})_{2}$, and $\mathrm{Pd}(\mathrm{dppf}) \mathrm{Cl}_{2}$ as the catalysts reduced the yield of the product (Table 1 , entries $2-4$ ). Although the temperature was increased to $110{ }^{\circ} \mathrm{C}$, almost no desired product was gained in DMF ( $N, N$-dimethyl formamide) (Table 1, entry 5). We observed that the main by-product of the reaction was the homocoupling of the arylboronic acid. Therefore, we increased the amount of $\mathbf{1 a}$ to two equivalents; however, the product 3a was obtained in almost the same yield (Table 1, entry 6 versus entry 1). Moreover, increasing the amount of $\mathrm{Na}_{2} \mathrm{CO}_{3}$ (6 equiv.) did not lead to a discernible effect on the yield (Table 1, entry 7 versus entry 1). We further optimized the reaction conditions by reducing the amount of $\mathrm{H}_{2} \mathrm{O}$, but the product was obtained in only $5 \%$ yield (Table 1, entry 8 ). For further improvement of the reaction, other bases such as $\mathrm{K}_{2} \mathrm{CO}_{3}, \mathrm{KF}$, and $\mathrm{Cs}_{2} \mathrm{CO}_{3}$ were also examined (Table 1, entries 9-11). To our delight, the product was obtained in $85 \%$ yield with $\mathrm{Cs}_{2} \mathrm{CO}_{3}$ as the base. Furthermore, we observed that an increase or reduction of the reaction temperature were both unfavorable for the transformation (Table 1, entries 12 and 13). When the amount of $\mathrm{Pd}\left(\mathrm{PPh}_{3}\right)_{4}$ was reduced to $5 \mathrm{~mol} \%$, the product $3 \mathbf{a}$ was obtained in a slightly lower yield (Table 1, entry 14). From the above-mentioned results, it was concluded that the optimized conditions for this Suzuki-Miyaura coupling reaction protocol were $10 \mathrm{~mol} \% \mathrm{Pd}\left(\mathrm{PPh}_{3}\right)_{4}, \mathrm{Cs}_{2} \mathrm{CO}_{3}$ (2 equiv.) in dioxane/ $\mathrm{H}_{2} \mathrm{O}(4: 1)$ at $90{ }^{\circ} \mathrm{C}$ (Table 1, entry 11); especially, we have observed no dehalogenation in the reaction. To assess the effect of SEM-protected and BOC-protected pyrroles, BOC-protected pyrrole (2a') was selected as the substrate. The reaction gave the product 3a in good yield, but the deprotected by-product (4a) was obtained in 5\% yield (Table 1, entry 15). The result was confirmed with the assay conducted using $\mathrm{Na}_{2} \mathrm{CO}_{3}$ as the base, which led to $4 \mathbf{a}$ in $11 \%$ yield (Table 1, entry 16). In conclusion, the SEM-protecting group was stable under the optimal reaction conditions compared to the BOC protecting group, and a cross-coupling product was obtained in very good yield.

Table 1. Screening reaction conditions of Suzuki coupling ${ }^{\mathrm{a}}$.

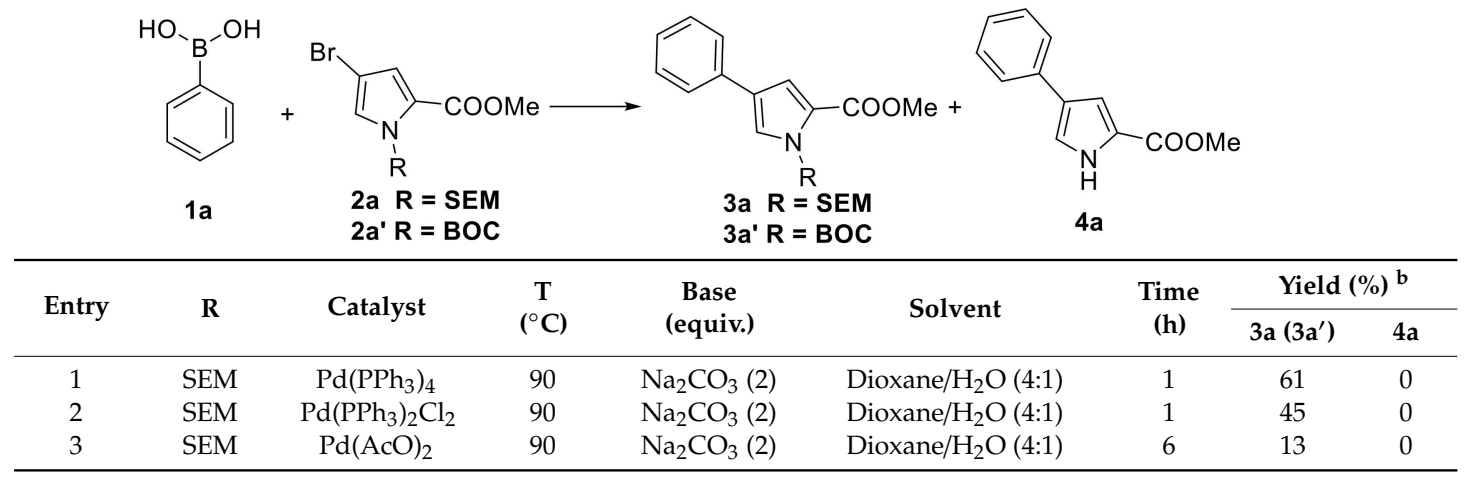


Table 1. Cont.

\begin{tabular}{|c|c|c|c|c|c|c|c|c|}
\hline \multirow{2}{*}{ Entry } & \multirow{2}{*}{$\mathbf{R}$} & \multirow{2}{*}{ Catalyst } & \multirow{2}{*}{$\begin{array}{c}\mathrm{T} \\
\left({ }^{\circ} \mathrm{C}\right)\end{array}$} & \multirow{2}{*}{$\begin{array}{c}\text { Base } \\
\text { (equiv.) }\end{array}$} & \multirow{2}{*}{ Solvent } & \multirow{2}{*}{$\begin{array}{l}\text { Time } \\
\text { (h) }\end{array}$} & \multicolumn{2}{|c|}{ Yield (\%) ${ }^{b}$} \\
\hline & & & & & & & $3 a\left(3 a^{\prime}\right)$ & $4 a$ \\
\hline 4 & SEM & $\mathrm{Pd}(\mathrm{dppf}) \mathrm{Cl}_{2}$ & 90 & $\mathrm{Na}_{2} \mathrm{CO}_{3}(2)$ & Dioxane $/ \mathrm{H}_{2} \mathrm{O}(4: 1)$ & 6 & 28 & 0 \\
\hline 5 & SEM & $\mathrm{Pd}\left(\mathrm{PPh}_{3}\right)_{4}$ & 110 & $\mathrm{Na}_{2} \mathrm{CO}_{3}(2)$ & DMF & 6 & trace & 0 \\
\hline $6^{c}$ & SEM & $\mathrm{Pd}\left(\mathrm{PPh}_{3}\right)_{4}$ & 90 & $\mathrm{Na}_{2} \mathrm{CO}_{3}(2)$ & Dioxane $/ \mathrm{H}_{2} \mathrm{O}(4: 1)$ & 1 & 57 & 0 \\
\hline $7^{c}$ & SEM & $\mathrm{Pd}\left(\mathrm{PPh}_{3}\right)_{4}$ & 90 & $\mathrm{Na}_{2} \mathrm{CO}_{3}(6)$ & Dioxane $/ \mathrm{H}_{2} \mathrm{O}(4: 1)$ & 1 & 57 & 0 \\
\hline 8 & SEM & $\mathrm{Pd}\left(\mathrm{PPh}_{3}\right)_{4}$ & 90 & $\mathrm{Na}_{2} \mathrm{CO}_{3}(2)$ & Dioxane $/ \mathrm{H}_{2} \mathrm{O}(4: 0.1)$ & 6 & 5 & 0 \\
\hline 9 & SEM & $\mathrm{Pd}\left(\mathrm{PPh}_{3}\right)_{4}$ & 90 & $\mathrm{~K}_{2} \mathrm{CO}_{3}(2)$ & Dioxane $/ \mathrm{H}_{2} \mathrm{O}(4: 1)$ & 5 & 45 & 0 \\
\hline 10 & SEM & $\mathrm{Pd}\left(\mathrm{PPh}_{3}\right)_{4}$ & 90 & KF (2) & Dioxane $/ \mathrm{H}_{2} \mathrm{O}(4: 1)$ & 6 & 32 & 0 \\
\hline 11 & SEM & $\mathrm{Pd}\left(\mathrm{PPh}_{3}\right)_{4}$ & 90 & $\mathrm{Cs}_{2} \mathrm{CO}_{3}(2)$ & Dioxane $/ \mathrm{H}_{2} \mathrm{O}(4: 1)$ & 5 & 85 & 0 \\
\hline 12 & SEM & $\mathrm{Pd}\left(\mathrm{PPh}_{3}\right)_{4}$ & 110 & $\mathrm{Cs}_{2} \mathrm{CO}_{3}(2)$ & Dioxane $/ \mathrm{H}_{2} \mathrm{O}(4: 1)$ & 3 & 75 & 0 \\
\hline 13 & SEM & $\mathrm{Pd}\left(\mathrm{PPh}_{3}\right)_{4}$ & 60 & $\mathrm{Cs}_{2} \mathrm{CO}_{3}(2)$ & Dioxane $/ \mathrm{H}_{2} \mathrm{O}(4: 1)$ & 5 & 53 & 0 \\
\hline $14^{\mathrm{d}}$ & SEM & $\mathrm{Pd}\left(\mathrm{PPh}_{3}\right)_{4}$ & 90 & $\mathrm{Cs}_{2} \mathrm{CO}_{3}(2)$ & Dioxane $/ \mathrm{H}_{2} \mathrm{O}(4: 1)$ & 2 & 78 & 0 \\
\hline 15 & BOC & $\mathrm{Pd}\left(\mathrm{PPh}_{3}\right)_{4}$ & 90 & $\mathrm{Cs}_{2} \mathrm{CO}_{3}(2)$ & Dioxane $/ \mathrm{H}_{2} \mathrm{O}(4: 1)$ & 2 & 76 & 5 \\
\hline 16 & BOC & $\mathrm{Pd}\left(\mathrm{PPh}_{3}\right)_{4}$ & 90 & $\mathrm{Na}_{2} \mathrm{CO}_{3}(2)$ & Dioxane $/ \mathrm{H}_{2} \mathrm{O}(4: 1)$ & 2 & 64 & 11 \\
\hline
\end{tabular}

a General conditions: 1a $(1.5 \mathrm{mmol}), 2 \mathrm{a}(1 \mathrm{mmol})$, catalyst $(10 \mathrm{~mol} \%)$, and base in $10 \mathrm{~mL}$ of the indicated solvent under $\mathrm{Ar}_{2}$ atmosphere. ${ }^{\mathrm{b}}$ Isolated yield. ${ }^{c}$ The equivalent ratio of $1 \mathrm{a}: 2 \mathrm{a}=2: 1 .{ }^{\mathrm{d}} 5 \mathrm{~mol} \% \mathrm{Pd}\left(\mathrm{PPh}_{3}\right)_{4}$.

With these conditions in hand, we next evaluated the substrate scope of the reaction (Scheme 1). Overall, it was found that all the attempted phenylboronic acid analogues produced the desired aryl-substituted pyrroles in moderate to excellent yields. To evaluate the effect of boronic acid substituents, we first studied the reaction of the substrate bearing the electron-withdrawing groups such as $\mathrm{Cl}, \mathrm{F}, \mathrm{NO}_{2}$, and $\mathrm{CF}_{3}(\mathbf{3 b}-\mathbf{i})$. When phenylboronic acid with $\mathrm{Cl}$ and $\mathrm{F}$ substituents were used in the ortho position, the target compounds ( $3 \mathbf{c}$ and $\mathbf{3 f}$ ) were isolated in $93 \%$ and $77 \%$ yield, respectively. In contrast, para substitution resulted in a decreased yield ( $3 \mathbf{b}$ and $\mathbf{3 e})$. The same behavior was observed with the 2,4-dichloro or 2,4-difluro phenylboronic acid ( $3 \mathrm{~d}$ and $3 \mathrm{~g}$ ). The presence of the strong electron-withdrawing $\mathrm{NO}_{2}$ and $\mathrm{CF}_{3}$ groups on the phenylboronic acid did not drastically affect the efficiency of the reaction ( $3 \mathbf{h}$ and $3 \mathbf{i})$.

Then, we focused our attention on the reactivity of the phenylboronic acid with the electron-donating groups. When the phenylboronic acid was substituted with an OMe group at the C-4 position, the reaction gave the product $3 \mathbf{j}$ in $77 \%$ yield, while the OMe in the ortho position afforded the desired product in $87 \%$ yield (3k). The results displayed that this reaction followed the same behavior as the cross-coupling reaction compared to 2-fluorophenylboronic acid and 2-chlorophenylboronic acid as the substrate. Nevertheless, the Me group in the ortho position of phenylboronic acid lowered the efficiency of the reaction ( 31 versus $3 \mathrm{~m}$ ). The reaction with the fused aromatic compounds gave the corresponding products in good yields as well (3n and 3o). Notably, the reaction can also be carried out successfully with the heterocyclic substrates to furnish the desired products (3p and $\mathbf{3 q}$ ). Finally, 5-bromopyrrole and 3-bromopyrrole ( $\mathbf{2} \mathbf{b}$ and $\mathbf{2 c})$ were chosen as the substrates to document the potential of the method. The reactions worked very smoothly, giving the corresponding products in excellent yields ( $3 \mathbf{r}$ and $3 \mathbf{s})$.

To further explore this synthetic potential, several common transformations of the product 3 a were conducted (Scheme 2). First, the SEM-protecting group was removed smoothly in the presence of TBAF (tetrabutyl ammonium fluoride) to provide the $\mathrm{N}-\mathrm{H}-4$-phenyl pyrrole $4 \mathrm{a}$ in $83 \%$ yield. Hydrolysis and reduction reactions readily occurred, leading to the fascinating pyrrole-2-carboxylic acid $4 \mathbf{b}$ and 2-hydroxymethyl substituted pyrrole compound $4 \mathrm{c}$ in good yields, respectively. No SEM deprotection of pyrrole was observed in the aforementioned hydrolysis and reduction reactions as anticipated. 


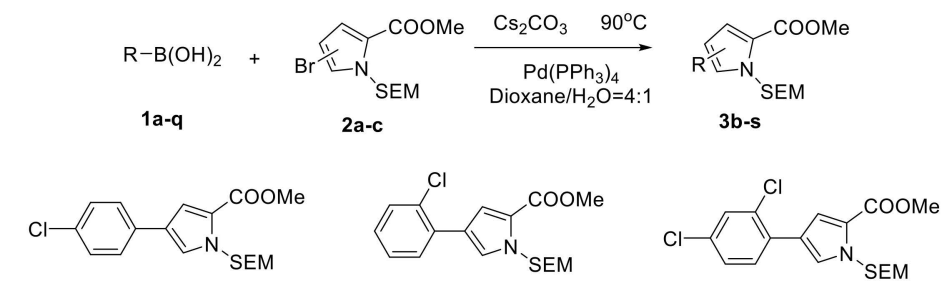

3b, $66 \%$

3c, $93 \%$

3d, $67 \%$

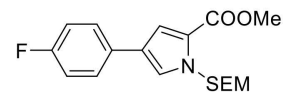

3e, $70 \%$<smiles>C#[Sn]n1cc(-c2ccc([N+](=O)[O-])cc2)cc1C(C)=O</smiles>

3h, $62 \%$

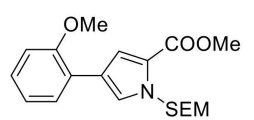

3k, $87 \%$

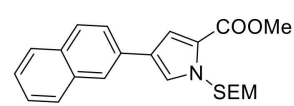

3n, $76 \%$

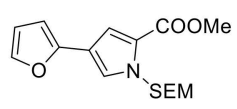

3q, $45 \%$

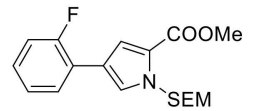

3f, $77 \%$

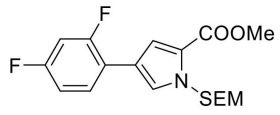

3g, $70 \%$

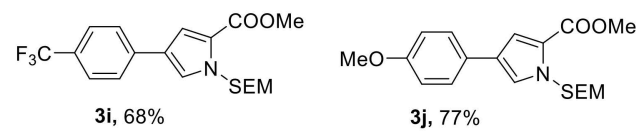

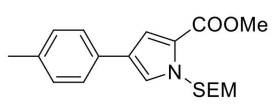

$31,73 \%$

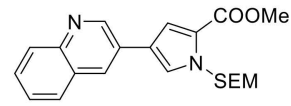

3o, $66 \%$

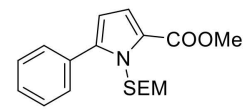

$3 r, 95 \%$

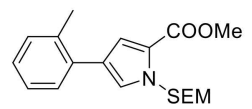

$3 m, 52 \%$

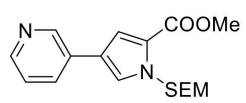

$3 p, 41 \%$

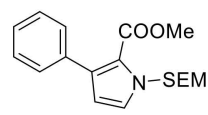

3s, $99 \%$

Scheme 1. Scope of the Suzuki coupling reaction. Yields refer to the isolated yields.

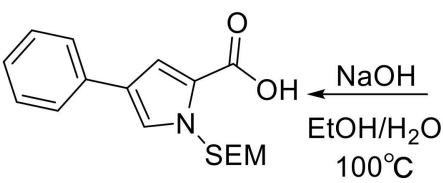

4b, $84 \%$<smiles>COC(=O)c1cc(-c2ccccc2)cn1[As]=[W]</smiles>

$3 a$<smiles>COC(=O)c1cc(-c2ccccc2)c[nH]1</smiles>

4a, $83 \%$<smiles>N#Cn1cc(-c2ccccc2)c(CO)c1CO</smiles>

4c, $89 \%$

Scheme 2. The application of SEM-protected pyrroles. Yields refer to the isolated yields.

\section{Materials and Methods}

\subsection{General Methods}

All the starting materials were obtained from commercial suppliers and were used without further purification unless stated otherwise. Reactions were monitored by Thin Layer Chromatography (silica gel GF254). Products were purified by column chromatography on silica gel (300-400 mesh). ${ }^{1} \mathrm{H}$ and ${ }^{13} \mathrm{C}$ spectra were recorded on a Varian (Palo Alto, CA, USA) 400 NMR or 500 NMR spectrometer using DMSO- $d_{6}$ as a solvent and tetramethylsilane (TMS) as an internal standard. Chemical shifts 
$(\delta)$ are reported in parts per million (ppm), and coupling constants $(J)$ are reported in Hertz $(\mathrm{Hz})$. Data are represented as follows: chemical shift, multiplicity $(\mathrm{br}=\mathrm{broad}, \mathrm{s}=$ singlet, $\mathrm{d}=\mathrm{doublet}$, $\mathrm{t}=$ triplet, $\mathrm{q}=$ quartet, $\mathrm{m}=$ multiplet), coupling constants in Hertz $(\mathrm{Hz})$, and integration. All the melting points were measured with a microscope melting point apparatus (MP-J3, Yanaco, Kyoto, Japan) and were uncorrected. High-resolution mass spectra were determined on ThermoExactive Orbitrap plus mass spectrometer (Waltham, MA, USA). ${ }^{1} \mathrm{H}-\mathrm{NMR}$ and HRMS spectra are available in Supplementary Materials.

\subsection{Typical Procedure to Synthesize Compounds $\mathbf{2 a - c}$}

First, $t$-BuOK (330 mg, $2.94 \mathrm{mmol}$ ) was added to a solution of bromopyrroles (500 mg, $2.45 \mathrm{mmol})$ in anhydrous DMF (5 mL) in an ice-bath, and the mixture was stirred for $0.5 \mathrm{~h}$. SEM-Cl (449 mg, $2.7 \mathrm{mmol}$ ) was added to the resulting mixture. The reaction mixture was stirred at $\mathrm{rt}$ for $2 \mathrm{~h}$, and then quenched with $\mathrm{H}_{2} \mathrm{O}$. The mixture was extracted with EtOAc $(15 \mathrm{~mL} \times 3)$, and the combined organic layer was washed with sat. $\mathrm{NaHCO}_{3}$ and brine, dried with $\mathrm{Na}_{2} \mathrm{SO}_{4}$, and concentrated. The product was obtained by flash chromatography (EtOAc in $\mathrm{PE}=3 \%$ ).

Methyl 4-bromo-1-((2-(trimethylsilyl)ethoxy)methyl)-1H-pyrrole-2-carboxylate (2a). Colorless oil, 71\% yield. ${ }^{1} \mathrm{H}-\mathrm{NMR}\left(400 \mathrm{MHz}, \mathrm{DMSO}-d_{6}\right) \delta: 7.50(\mathrm{~d}, J=2.0 \mathrm{~Hz}, 1 \mathrm{H}$, Pyrrole-5H), $6.94(\mathrm{~d}, J=2.0 \mathrm{~Hz}, 1 \mathrm{H}, \mathrm{Pyrrole}-3 \mathrm{H})$, $5.58\left(\mathrm{~s}, 2 \mathrm{H}, \mathrm{NCH}_{2} \mathrm{O}\right), 3.74\left(\mathrm{~s}, 3 \mathrm{H}, \mathrm{CH}_{3}\right), 3.46\left(\mathrm{t}, J=8.0 \mathrm{~Hz}, 2 \mathrm{H}, \mathrm{OCH}_{2} \mathrm{CH}_{2} \mathrm{Si}\right), 0.79(\mathrm{t}, J=8.0 \mathrm{~Hz}, 2 \mathrm{H}$, $\left.\mathrm{OCH}_{2} \mathrm{CH}_{2} \mathrm{Si}\right),-0.07$ (s, 9H, $\left.\mathrm{Si}\left(\mathrm{CH}_{3}\right)_{3}\right)$. HRMS (ESI): $m / z[\mathrm{M}+\mathrm{H}]^{+}$calcd for $\mathrm{C}_{12} \mathrm{H}_{21} \mathrm{BrNO}_{3} \mathrm{Si}: 334.0469$; found: 334.0448 .

Methyl 5-bromo-1-((2-(trimethylsilyl)ethoxy)methyl)-1H-pyrrole-2-carboxylate (2b). Colorless oil, 96\% yield. ${ }^{1} \mathrm{H}-\mathrm{NMR}\left(400 \mathrm{MHz}, \mathrm{DMSO}-\mathrm{d}_{6}\right) \delta: 6.99(\mathrm{~d}, J=4.0 \mathrm{~Hz}, 1 \mathrm{H}$, Pyrrole-3H), $6.40(\mathrm{~d}, J=4.0 \mathrm{~Hz}, 1 \mathrm{H}, \mathrm{Pyrrole}-4 \mathrm{H})$, $5.71\left(\mathrm{~s}, 2 \mathrm{H}, \mathrm{NCH}_{2} \mathrm{O}\right), 3.75\left(\mathrm{~s}, 3 \mathrm{H}, \mathrm{CH}_{3}\right), 3.51\left(\mathrm{t}, \mathrm{J}=8.0 \mathrm{~Hz}, 2 \mathrm{H}, \mathrm{OCH}_{2} \mathrm{CH}_{2} \mathrm{Si}\right), 0.80(\mathrm{t}, J=8.0 \mathrm{~Hz}, 2 \mathrm{H}$, $\left.\mathrm{OCH}_{2} \mathrm{CH}_{2} \mathrm{Si}\right),-0.07$ (s, 9H, $\left.\mathrm{Si}\left(\mathrm{CH}_{3}\right)_{3}\right)$. HRMS (ESI): $m / z[\mathrm{M}+\mathrm{H}]^{+}$calcd for $\mathrm{C}_{12} \mathrm{H}_{21} \mathrm{BrNO}_{3} \mathrm{Si}: 334.0469$; found: 334.0463 .

Methyl 3-bromo-1-((2-(trimethylsilyl)ethoxy)methyl)-1H-pyrrole-2-carboxylate (2c). Colorless oil, 81\% yield. ${ }^{1} \mathrm{H}-\mathrm{NMR}\left(500 \mathrm{MHz}, \mathrm{DMSO}-d_{6}\right) \delta: 7.33(\mathrm{~d}, J=2.5 \mathrm{~Hz}, 1 \mathrm{H}$, Pyrrole- $5 \mathrm{H}), 6.35(\mathrm{~d}, J=2.5 \mathrm{~Hz}, 1 \mathrm{H}, \mathrm{Pyrrole}-4 \mathrm{H})$, $5.57\left(\mathrm{~s}, 2 \mathrm{H}, \mathrm{NCH}_{2} \mathrm{O}\right), 3.78\left(\mathrm{~s}, 3 \mathrm{H}, \mathrm{CH}_{3}\right), 3.41\left(\mathrm{t}, \mathrm{J}=7.5 \mathrm{~Hz}, 2 \mathrm{H}, \mathrm{OCH}_{2} \mathrm{CH}_{2} \mathrm{Si}\right), 0.78(\mathrm{t}, J=7.5 \mathrm{~Hz}, 2 \mathrm{H}$, $\left.\mathrm{OCH}_{2} \mathrm{CH}_{2} \mathrm{Si}\right),-0.08\left(\mathrm{~s}, 9 \mathrm{H}, \mathrm{Si}\left(\mathrm{CH}_{3}\right)_{3}\right)$. HRMS (ESI): $m / z[\mathrm{M}+\mathrm{H}]^{+}$calcd for $\mathrm{C}_{12} \mathrm{H}_{21} \mathrm{BrNO}_{3} \mathrm{Si}: 334.0469$; found: 334.0460 .

\subsection{Typical Procedure to Synthesize Products $\mathbf{3 a - s}$}

Dioxane $(8 \mathrm{~mL})$ and $\mathrm{H}_{2} \mathrm{O}(2 \mathrm{~mL})$ were added to the mixture of $\mathbf{1 a}-\mathbf{q}(1.5 \mathrm{mmol}), \mathbf{2} \mathbf{a}-\mathbf{c}(1 \mathrm{mmol})$, $\mathrm{Cs}_{2} \mathrm{CO}_{3}(652 \mathrm{mg}, 2 \mathrm{mmol})$, and $\mathrm{Pd}\left(\mathrm{PPh}_{3}\right)_{4}(116 \mathrm{mg}, 0.1 \mathrm{mmol})$ under $\mathrm{Ar}_{2}$ atmosphere. The reaction mixture was stirred at $90^{\circ} \mathrm{C}$ for about $5 \mathrm{~h}$ (monitored by Thin Layer Chromatography) and cooled to rt. Then, the mixture was filtered to remove the solids, and the filtrate was concentrated. Purification by flash chromatography (EtOAc in $\mathrm{PE}=3 \%$ ) gave the desired product.

Methyl 4-phenyl-1-((2-(trimethylsilyl)ethoxy)methyl)-1H-pyrrole-2-carboxylate (3a). Colorless oil, 85\% yield. ${ }^{1} \mathrm{H}-\mathrm{NMR}\left(400 \mathrm{MHz}, \mathrm{DMSO}-\mathrm{d}_{6}\right) \delta: 7.80(\mathrm{~d}, J=1.2 \mathrm{~Hz}, 1 \mathrm{H}$, Pyrrole-5H), $7.61(\mathrm{~d}, J=8.0 \mathrm{~Hz}, 2 \mathrm{H}, \mathrm{Ar}-2,6 \mathrm{H})$, 7.36-7.32 (m, 3H, Ar-3,5H, Pyrrole-3H), 7.19 (t, J = 8.0 Hz, 1H, Ar-4H), $5.64\left(\mathrm{~s}, 2 \mathrm{H}, \mathrm{NCH}_{2} \mathrm{O}\right), 3.77$ (s, 3H, $\left.\mathrm{CH}_{3}\right), 3.50\left(\mathrm{t}, J=7.6 \mathrm{~Hz}, 2 \mathrm{H}, \mathrm{OCH}_{2} \mathrm{CH}_{2} \mathrm{Si}\right), 0.82\left(\mathrm{t}, J=7.6 \mathrm{~Hz}, 2 \mathrm{H}, \mathrm{OCH}_{2} \mathrm{CH}_{2} \mathrm{Si}\right),-0.07\left(\mathrm{~s}, 9 \mathrm{H}, \mathrm{Si}\left(\mathrm{CH}_{3}\right)_{3}\right)$. ${ }^{13} \mathrm{C}-\mathrm{NMR}\left(100 \mathrm{MHz}\right.$, DMSO- $\left.d_{6}\right) \delta: 161.0,134.2,129.2,127.4,126.6,125.2,123.9,122.7,116.1,76.9,65.6$, 51.6, 17.6, -1.0. HRMS (ESI): $m / z[\mathrm{M}+\mathrm{H}]^{+}$calcd for $\mathrm{C}_{18} \mathrm{H}_{26} \mathrm{NO}_{3} \mathrm{Si}: 332.1677$; found: 332.1662 .

Methyl 4-(4-chlorophenyl)-1-((2-(trimethylsilyl)ethoxy)methyl)-1H-pyrrole-2-carboxylate (3b). Colorless oil, 66\% yield. ${ }^{1} \mathrm{H}-\mathrm{NMR}\left(500 \mathrm{MHz}, \mathrm{DMSO}-d_{6}\right) \delta: 7.84(\mathrm{~s}, 1 \mathrm{H}$, Pyrrole-5H), 7.65 (d, J = 8.5 Hz, 2H, Ar-2,6H), 7.39 (d, $J=8.5$ Hz, 2H, Ar-3,5H), 7.35 (s, 1H, Pyrrole-3H), 5.64 (s, 2H, NCH $\mathrm{NC}_{2}, 3.77\left(\mathrm{~s}, 3 \mathrm{H}, \mathrm{CH}_{3}\right), 3.50$ $\left(\mathrm{t}, J=8.0 \mathrm{~Hz}, 2 \mathrm{H}, \mathrm{OCH}_{2} \mathrm{CH}_{2} \mathrm{Si}\right), 0.82\left(\mathrm{t}, \mathrm{J}=8.0 \mathrm{~Hz}, 2 \mathrm{H}, \mathrm{OCH}_{2} \mathrm{CH}_{2} \mathrm{Si}\right),-0.07\left(\mathrm{~s}, 9 \mathrm{H}, \mathrm{Si}\left(\mathrm{CH}_{3}\right)_{3}\right) .{ }^{13} \mathrm{C}-\mathrm{NMR}$ 
$\left(100 \mathrm{MHz}, \mathrm{DMSO}-d_{6}\right) \delta: 160.9,133.2,130.9,129.2,127.7,126.9,122.9,122.6,116.2,76.9,65.6,51.7,17.5$, -1.0. HRMS (ESI): $\mathrm{m} / z$ [M + H] ${ }^{+}$calcd for $\mathrm{C}_{18} \mathrm{H}_{25} \mathrm{ClNO}_{3} \mathrm{Si}$ : 366.1287 ; found: 366.1271 .

Methyl 4-(2-chlorophenyl)-1-((2-(trimethylsilyl)ethoxy)methyl)-1H-pyrrole-2-carboxylate (3c). Colorless oil, 93\% yield. ${ }^{1} \mathrm{H}-\mathrm{NMR}\left(500 \mathrm{MHz}\right.$, DMSO- $\left.d_{6}\right) \delta: 7.75(\mathrm{~s}, 1 \mathrm{H}$, Pyrrole- $5 \mathrm{H}), 7.56(\mathrm{~d}, J=7.5 \mathrm{~Hz}, 1 \mathrm{H}, \mathrm{Ar}-3 \mathrm{H})$, $7.50(\mathrm{~d}, J=7.5 \mathrm{~Hz}, 1 \mathrm{H}, \mathrm{Ar}-6 \mathrm{H}), 7.36(\mathrm{t}, J=7.0 \mathrm{~Hz}, 1 \mathrm{H}, \mathrm{Ar}-5 \mathrm{H}), 7.29-7.27(\mathrm{~m}, 2 \mathrm{H}$, Ar- $4 \mathrm{H}$, Pyrrole- $3 \mathrm{H})$, $5.68\left(\mathrm{~s}, 2 \mathrm{H}, \mathrm{NCH}_{2} \mathrm{O}\right), 3.77\left(\mathrm{~s}, 3 \mathrm{H}, \mathrm{CH}_{3}\right), 3.52\left(\mathrm{t}, J=7.5 \mathrm{~Hz}, 2 \mathrm{H}, \mathrm{OCH}_{2} \mathrm{CH}_{2} \mathrm{Si}\right), 0.82(\mathrm{t}, J=7.5 \mathrm{~Hz}, 2 \mathrm{H}$, $\left.\mathrm{OCH}_{2} \mathrm{CH}_{2} \mathrm{Si}\right),-0.07$ (s, 9H, $\left.\mathrm{Si}\left(\mathrm{CH}_{3}\right)_{3}\right) .{ }^{13} \mathrm{C}-\mathrm{NMR}\left(100 \mathrm{MHz}, \mathrm{DMSO}-d_{6}\right) \delta: 160.9,133.0,131.1,130.7$, 130.0, 128.5, 128.0, 121.9, 120.8, 119.1, 77.0, 65.6, 51.7, 17.6, -1.0. HRMS (ESI): $m / z$ [M + H] ${ }^{+}$calcd for $\mathrm{C}_{18} \mathrm{H}_{25} \mathrm{ClNO}_{3} \mathrm{Si}$ : 366.1287; found: 366.1274 .

Methyl 4-(2,4-dichlorophenyl)-1-((2-(trimethylsilyl)ethoxy)methyl)-1H-pyrrole-2-carboxylate (3d). Colorless oil, $67 \%$ yield. ${ }^{1} \mathrm{H}-\mathrm{NMR}\left(500 \mathrm{MHz}\right.$, DMSO- $\left.d_{6}\right) \delta 7.78(\mathrm{~d}, J=1.5 \mathrm{~Hz}, 1 \mathrm{H}$, Pyrrole- $5 \mathrm{H}), 7.65(\mathrm{~d}, J=1.5 \mathrm{~Hz}$, $1 \mathrm{H}, \mathrm{Ar}-3 \mathrm{H}), 7.60(\mathrm{~d}, J=8.5 \mathrm{~Hz}, 1 \mathrm{H}, \mathrm{Ar}-6 \mathrm{H}), 7.43(\mathrm{dd}, J=8.0,1.5 \mathrm{~Hz}, 1 \mathrm{H}, \mathrm{Ar}-5 \mathrm{H}), 7.28(\mathrm{~d}, J=1.5 \mathrm{~Hz}, 1 \mathrm{H}$, Pyrrole-3H), $5.68\left(\mathrm{~s}, 2 \mathrm{H}, \mathrm{NCH}_{2} \mathrm{O}\right), 3.78\left(\mathrm{~s}, 3 \mathrm{H}, \mathrm{CH}_{3}\right), 3.52\left(\mathrm{t}, J=8.0 \mathrm{~Hz}, 2 \mathrm{H}, \mathrm{OCH}_{2} \mathrm{CH}_{2} \mathrm{Si}\right), 0.82(\mathrm{t}, J=8.0$ $\left.\mathrm{Hz}, 2 \mathrm{H}, \mathrm{OCH}_{2} \mathrm{CH}_{2} \mathrm{Si}\right),-0.07\left(\mathrm{~s}, 9 \mathrm{H}, \mathrm{Si}\left(\mathrm{CH}_{3}\right)_{3}\right) .{ }^{13} \mathrm{C}-\mathrm{NMR}\left(100 \mathrm{MHz}, \mathrm{DMSO}-d_{6}\right) \delta: 160.8,132.0,131.9$, $131.8,130.1,130.0,128.1,122.0,119.7,119.0,77.0,65.6,51.7,17.6,-1.0$. HRMS (ESI): $m / z$ [M + H] ${ }^{+}$calcd for $\mathrm{C}_{18} \mathrm{H}_{24} \mathrm{Cl}_{2} \mathrm{NO}_{3} \mathrm{Si}: 400.0897$; found: 400.0892 .

Methyl 4-(4-fluorophenyl)-1-((2-(trimethylsilyl)ethoxy)methyl)-1H-pyrrole-2-carboxylate (3e). Colorless oil, $70 \%$ yield. ${ }^{1} \mathrm{H}-\mathrm{NMR}\left(400 \mathrm{MHz}, \mathrm{DMSO}-d_{6}\right) \delta: 7.77(\mathrm{~d}, J=2.0 \mathrm{~Hz}, 1 \mathrm{H}$, Pyrrole- $5 \mathrm{H}), 7.66-7.63(\mathrm{~m}, 2 \mathrm{H}$, Ar-2,6H), $7.31(\mathrm{~d}, J=2.0 \mathrm{~Hz}, 1 \mathrm{H}$, Pyrrole- $3 \mathrm{H}), 7.17(\mathrm{t}, J=8.8 \mathrm{~Hz}, 2 \mathrm{H}, \mathrm{Ar}-3,5 \mathrm{H}), 5.63\left(\mathrm{~s}, 2 \mathrm{H}, \mathrm{NCH}_{2} \mathrm{O}\right)$, $3.77\left(\mathrm{~s}, 3 \mathrm{H}, \mathrm{CH}_{3}\right), 3.50\left(\mathrm{t}, J=8.0 \mathrm{~Hz}, 2 \mathrm{H}, \mathrm{OCH}_{2} \mathrm{CH}_{2} \mathrm{Si}\right), 0.81\left(\mathrm{t}, J=8.0 \mathrm{~Hz}, 2 \mathrm{H}, \mathrm{OCH}_{2} \mathrm{CH}_{2} \mathrm{Si}\right),-0.08(\mathrm{~s}$, $\left.9 \mathrm{H}, \mathrm{Si}\left(\mathrm{CH}_{3}\right)_{3}\right) .{ }^{13} \mathrm{C}-\mathrm{NMR}\left(100 \mathrm{MHz}, \mathrm{DMSO}-d_{6}\right) \delta: 161.2(\mathrm{~d}, J=241.1 \mathrm{~Hz}), 160.9,130.8(\mathrm{~d}, J=2.9 \mathrm{~Hz})$, $127.3,127.1(\mathrm{~d}, J=7.8 \mathrm{~Hz}), 122.8(\mathrm{~d}, J=23.6 \mathrm{~Hz}), 116.1,116.0,115.9,76.9,65.6,51.6,17.6,-1.0$. HRMS (ESI): $m / z[\mathrm{M}+\mathrm{H}]^{+}$calcd for $\mathrm{C}_{18} \mathrm{H}_{25} \mathrm{FNO}_{3} \mathrm{Si}$ : 350.1582; found: 350.1565 .

Methyl 4-(2-fluorophenyl)-1-((2-(trimethylsilyl)ethoxy)methyl)-1H-pyrrole-2-carboxylate (3f). Colorless oil, 77\% yield. ${ }^{1} \mathrm{H}-\mathrm{NMR}\left(500 \mathrm{MHz}, \mathrm{DMSO}-d_{6}\right) \delta: 7.76-7.72(\mathrm{~m}, 2 \mathrm{H}$, Pyrrole-5H, Ar-6H), $7.36(\mathrm{~s}, 1 \mathrm{H}$, Pyrrole-3H), 7.26-7.21 (m, 3H, Ar-3,4,5H), $5.68\left(\mathrm{~s}, 2 \mathrm{H}, \mathrm{NCH}_{2} \mathrm{O}\right), 3.78\left(\mathrm{~s}, 3 \mathrm{H}, \mathrm{CH}_{3}\right), 3.51(\mathrm{t}, J=7.5 \mathrm{~Hz}$, $\left.2 \mathrm{H}, \mathrm{OCH}_{2} \mathrm{CH}_{2} \mathrm{Si}\right), 0.81\left(\mathrm{t}, J=7.5 \mathrm{~Hz}, 2 \mathrm{H}, \mathrm{OCH}_{2} \mathrm{CH}_{2} \mathrm{Si}\right),-0.07\left(\mathrm{~s}, 9 \mathrm{H}, \mathrm{Si}\left(\mathrm{CH}_{3}\right)_{3}\right) .{ }^{13} \mathrm{C}-\mathrm{NMR}(100 \mathrm{MHz}$, DMSO- $\left.d_{6}\right) \delta: 160.9,159.3(\mathrm{~d}, J=244.4 \mathrm{~Hz}), 129.3(\mathrm{~d}, J=8.6 \mathrm{~Hz}), 128.3(\mathrm{~d}, J=4.2 \mathrm{~Hz}), 128.2(\mathrm{~d}, J=8.4$ $\mathrm{Hz}), 125.2(\mathrm{~d}, J=3.1 \mathrm{~Hz}), 122.4,121.8(\mathrm{~d}, J=12.8 \mathrm{~Hz}), 117.5,117.4,116.5(\mathrm{~d}, J=22.1 \mathrm{~Hz}), 76.9,65.6,51.7$, 17.5, -1.0. HRMS (ESI): $\mathrm{m} / z$ [M + H] $]^{+}$calcd for $\mathrm{C}_{18} \mathrm{H}_{25} \mathrm{FNO}_{3} \mathrm{Si}$ : 350.1582; found: 350.1572 .

Methyl 4-(2,4-difluorophenyl)-1-((2-(trimethylsilyl)ethoxy)methyl)-1H-pyrrole-2-carboxylate (3g). Colorless oil, 70\% yield. ${ }^{1} \mathrm{H}-\mathrm{NMR}\left(500 \mathrm{MHz}, \mathrm{DMSO}-d_{6}\right) \delta: 7.78(\mathrm{dd}, J=16.0,9.0 \mathrm{~Hz}, 1 \mathrm{H}, \mathrm{Ar}-3 \mathrm{H}), 7.74(\mathrm{~s}, 1 \mathrm{H}$, Pyrrole-5H), $7.34(\mathrm{~s}, 1 \mathrm{H}$, Pyrrole-3H), $7.28(\mathrm{t}, J=9.5 \mathrm{~Hz}, 1 \mathrm{H}, \mathrm{Ar}-6 \mathrm{H}), 7.10(\mathrm{t}, J=8.5 \mathrm{~Hz}, 1 \mathrm{H}, \mathrm{Ar}-5 \mathrm{H})$, $5.68\left(\mathrm{~s}, 2 \mathrm{H}, \mathrm{NCH}_{2} \mathrm{O}\right), 3.79\left(\mathrm{~s}, 3 \mathrm{H}, \mathrm{CH}_{3}\right), 3.51\left(\mathrm{t}, J=8.0 \mathrm{~Hz}, 2 \mathrm{H}, \mathrm{OCH}_{2} \mathrm{CH}_{2} \mathrm{Si}\right), 0.82(\mathrm{t}, J=8.0 \mathrm{~Hz}, 2 \mathrm{H}$, $\left.\mathrm{OCH}_{2} \mathrm{CH}_{2} \mathrm{Si}\right),-0.07\left(\mathrm{~s}, 9 \mathrm{H}, \mathrm{Si}\left(\mathrm{CH}_{3}\right)_{3}\right) .{ }^{13} \mathrm{C}-\mathrm{NMR}\left(100 \mathrm{MHz}, \mathrm{DMSO}-d_{6}\right) \delta: 161.1(\mathrm{dd}, J=244.0,12.2 \mathrm{~Hz})$, $160.8,159.3(\mathrm{dd}, J=247.3,11.9 \mathrm{~Hz}), 129.4(\mathrm{dd}, J=9.3,5.9 \mathrm{~Hz}), 129.0(\mathrm{~d}, J=8.5 \mathrm{~Hz}), 122.4,118.5(\mathrm{dd}, J=$ 13.0, 3.6 Hz), $117.3(\mathrm{~d}, J=3.5 \mathrm{~Hz}), 116.8,112.3(\mathrm{dd}, J=21.0,3.2 \mathrm{~Hz}), 104.8(\mathrm{t}, J=26.1 \mathrm{~Hz}), 76.9,65.6,51.6$, 17.5, -1.1. HRMS (ESI): $\mathrm{m} / \mathrm{z}[\mathrm{M}+\mathrm{H}]^{+}$calcd for $\mathrm{C}_{18} \mathrm{H}_{24} \mathrm{~F}_{2} \mathrm{NO}_{3} \mathrm{Si}$ : 368.1488 ; found: 368.1480 .

Methyl 4-(4-nitrophenyl)-1-((2-(trimethylsilyl)ethoxy)methyl)-1H-pyrrole-2-carboxylate (3h). Yellow solid, $62 \%$ yield. M.p. $82-83^{\circ} \mathrm{C} .{ }^{1} \mathrm{H}-\mathrm{NMR}\left(400 \mathrm{MHz}, \mathrm{DMSO}-d_{6}\right) \delta: 8.20(\mathrm{~d}, J=8.4 \mathrm{~Hz}, 2 \mathrm{H}, \mathrm{Ar}-3,5 \mathrm{H}), 8.08$ $(\mathrm{d}, J=1.6 \mathrm{~Hz}, 1 \mathrm{H}$, Pyrrole- $5 \mathrm{H}), 7.91(\mathrm{~d}, J=8.4 \mathrm{~Hz}, 2 \mathrm{H}, \mathrm{Ar}-2,6 \mathrm{H}), 7.51(\mathrm{~d}, J=2.0 \mathrm{~Hz}, 1 \mathrm{H}$, Pyrrole- $3 \mathrm{H})$, $5.66\left(\mathrm{~s}, 2 \mathrm{H}, \mathrm{NCH}_{2} \mathrm{O}\right), 3.79\left(\mathrm{~s}, 3 \mathrm{H}, \mathrm{CH}_{3}\right), 3.52\left(\mathrm{t}, J=8.0 \mathrm{~Hz}, 2 \mathrm{H}, \mathrm{OCH}_{2} \mathrm{CH}_{2} \mathrm{Si}\right), 0.83(\mathrm{t}, J=8.0 \mathrm{~Hz}, 2 \mathrm{H}$, $\left.\mathrm{OCH}_{2} \mathrm{CH}_{2} \mathrm{Si}\right),-0.07\left(\mathrm{~s}, 9 \mathrm{H}, \mathrm{Si}\left(\mathrm{CH}_{3}\right)_{3}\right) .{ }^{13} \mathrm{C}-\mathrm{NMR}\left(100 \mathrm{MHz}, \mathrm{DMSO}-d_{6}\right) \delta: 160.8,145.7,141.3,129.4,125.8$, 124.7, 123.5, 121.8, 116.8, 77.2, 65.8, 51.8, 17.5, -1.0. HRMS (ESI): $\mathrm{m} / z[\mathrm{M}+\mathrm{H}]^{+}$calcd for $\mathrm{C}_{18} \mathrm{H}_{25} \mathrm{~N}_{2} \mathrm{O}_{5} \mathrm{Si}$ : 377.1527; found: 377.1545 .

Methyl 4-(4-(trifluoromethyl)phenyl)-1-((2-(trimethylsilyl)ethoxy)methyl)-1H-pyrrole-2-carboxylate (3i). Colorless oil, $68 \%$ yield. ${ }^{1} \mathrm{H}-\mathrm{NMR}\left(500 \mathrm{MHz}\right.$, DMSO- $\left.d_{6}\right) \delta: 7.95(\mathrm{~s}, 1 \mathrm{H}$, Pyrrole- $5 \mathrm{H}), 7.83(\mathrm{~d}, J=$ 
$5.0 \mathrm{~Hz}, 2 \mathrm{H}, \mathrm{Ar}-3,5 \mathrm{H}), 7.67$ (brs, 2H, Ar-2, 6H), 7.43 (s, 1H, Pyrrole- $3 \mathrm{H}), 5.65$ (s, 2H, NCH $\mathrm{N}_{2} \mathrm{O}$ ), 3.78 (s, $\left.3 \mathrm{H}, \mathrm{CH}_{3}\right), 3.51\left(\mathrm{t}, J=7.5 \mathrm{~Hz}, 2 \mathrm{H}, \mathrm{OCH}_{2} \mathrm{CH}_{2} \mathrm{Si}\right), 0.82\left(\mathrm{t}, J=7.5 \mathrm{~Hz}, 2 \mathrm{H}, \mathrm{OCH}_{2} \mathrm{CH}_{2} \mathrm{Si}\right),-0.08(\mathrm{~s}, 9 \mathrm{H}$, $\left.\mathrm{Si}\left(\mathrm{CH}_{3}\right)_{3}\right) .{ }^{13} \mathrm{C}-\mathrm{NMR}\left(100 \mathrm{MHz}, \mathrm{DMSO}-d_{6}\right) \delta: 160.9,138.4,128.5,126.9,126.1(\mathrm{q}, J=3.7 \mathrm{~Hz}), 125.6,125.1$ $(\mathrm{q}, J=236.0 \mathrm{~Hz}), 123.2,122.3,116.5,77.1,65.7,51.7,17.5,-1.0$. HRMS (ESI): $\mathrm{m} / z[\mathrm{M}+\mathrm{H}]^{+}$calcd for $\mathrm{C}_{19} \mathrm{H}_{25} \mathrm{~F}_{3} \mathrm{NO}_{3} \mathrm{Si}$ : 400.1550; found: 400.1567.

Methyl 4-(4-methoxyphenyl)-1-((2-(trimethylsilyl)ethoxy)methyl)-1H-pyrrole-2-carboxylate (3j). Colorless oil, $77 \%$ yield. ${ }^{1} \mathrm{H}-\mathrm{NMR}\left(400 \mathrm{MHz}, \mathrm{DMSO}-d_{6}\right) \delta: 7.68(\mathrm{~d}, J=1.6 \mathrm{~Hz}, 1 \mathrm{H}$, Pyrrole-5H), $7.53(\mathrm{~d}, J=8.8 \mathrm{~Hz}$, $2 \mathrm{H}, \mathrm{Ar}-2,6 \mathrm{H}), 7.25(\mathrm{~d}, J=2.0 \mathrm{~Hz}, 1 \mathrm{H}$, Pyrrole- $3 \mathrm{H}), 6.92(\mathrm{~d}, J=8.8 \mathrm{~Hz}, 2 \mathrm{H}, \mathrm{Ar}-3,5 \mathrm{H}), 5.63\left(\mathrm{~s}, 2 \mathrm{H}, \mathrm{NCH}_{2} \mathrm{O}\right)$, $3.76\left(\mathrm{~s}, 3 \mathrm{H}, \mathrm{CH}_{3}\right), 3.75\left(\mathrm{~s}, 3 \mathrm{H}, \mathrm{CH}_{3}\right), 3.50\left(\mathrm{t}, J=8.0 \mathrm{~Hz}, 2 \mathrm{H}, \mathrm{OCH}_{2} \mathrm{CH}_{2} \mathrm{Si}\right), 0.81(\mathrm{t}, J=8.0 \mathrm{~Hz}, 2 \mathrm{H}$, $\left.\mathrm{OCH}_{2} \mathrm{CH}_{2} \mathrm{Si}\right),-0.07\left(\mathrm{~s}, 9 \mathrm{H}, \mathrm{Si}\left(\mathrm{CH}_{3}\right)_{3}\right) .{ }^{13} \mathrm{C}-\mathrm{NMR}\left(100 \mathrm{MHz}, \mathrm{DMSO}-d_{6}\right) \delta: 161.0,158.3,126.8,126.6$, 126.4, 123.8, 122.5, 115.8, 114.6, 76.8, 65.5, 55.5, 51.5, 17.6, -1.0. HRMS (ESI): $m / z$ [M + H] ${ }^{+}$calcd for $\mathrm{C}_{19} \mathrm{H}_{28} \mathrm{NO}_{4} \mathrm{Si}$ : 362.1782 ; found: 362.1792 .

Methyl 4-(2-methoxyphenyl)-1-((2-(trimethylsilyl)ethoxy)methyl)-1H-pyrrole-2-carboxylate (3k). Colorless oil, 87\% yield. ${ }^{1} \mathrm{H}-\mathrm{NMR}\left(400 \mathrm{MHz}\right.$, DMSO- $\left.d_{6}\right) \delta: 7.76(\mathrm{~d}, J=2.0 \mathrm{~Hz}, 1 \mathrm{H}$, Pyrrole-5H), 7.59 (dd, $J=8.0$, $2.0 \mathrm{~Hz}, 1 \mathrm{H}, \mathrm{Ar}-6 \mathrm{H}), 7.37$ (d, J = 2.0 Hz, 1H, Pyrrole-3H), 7.20 (td, J = 7.6, 1.6 Hz, 1H, Ar-4H), 7.05 (d, J = $7.6 \mathrm{~Hz}, 1 \mathrm{H}, \mathrm{Ar}-3 \mathrm{H}), 6.95(\mathrm{td}, J=7.6,1.2 \mathrm{~Hz}, 1 \mathrm{H}, \mathrm{Ar}-5 \mathrm{H}), 5.67\left(\mathrm{~s}, 2 \mathrm{H}, \mathrm{NCH}_{2} \mathrm{O}\right), 3.86\left(\mathrm{~s}, 3 \mathrm{H}, \mathrm{CH}_{3}\right), 3.77$ $\left(\mathrm{s}, 3 \mathrm{H}, \mathrm{CH}_{3}\right), 3.51\left(\mathrm{t}, J=7.6 \mathrm{~Hz}, 2 \mathrm{H}, \mathrm{OCH}_{2} \mathrm{CH}_{2} \mathrm{Si}\right), 0.82\left(\mathrm{t}, J=7.6 \mathrm{~Hz}, 2 \mathrm{H}, \mathrm{OCH}_{2} \mathrm{CH}_{2} \mathrm{Si}\right),-0.06(\mathrm{~s}, 9 \mathrm{H}$, $\left.\mathrm{Si}\left(\mathrm{CH}_{3}\right)_{3}\right) .{ }^{13} \mathrm{C}-\mathrm{NMR}\left(100 \mathrm{MHz}, \mathrm{DMSO}-d_{6}\right) \delta: 161.1,156.3,129.9,127.7,122.6,121.6,121.2,120.0,118.0$, 112.1, 76.7, 65.4, 55.8, 51.5, 17.6, -1.0. HRMS (ESI): $m / z[\mathrm{M}+\mathrm{H}]^{+}$calcd for $\mathrm{C}_{19} \mathrm{H}_{28} \mathrm{NO}_{4} \mathrm{Si}: 362.1782$; found: 362.1784 .

Methyl 4-(p-tolyl)-1-((2-(trimethylsilyl)ethoxy)methyl)-1H-pyrrole-2-carboxylate (31). Colorless oil, 73\% yield. ${ }^{1} \mathrm{H}-\mathrm{NMR}\left(400 \mathrm{MHz}\right.$, DMSO- $\left.d_{6}\right) \delta: 7.74(\mathrm{~d}, J=2.0 \mathrm{~Hz}, 1 \mathrm{H}$, Pyrrole- $5 \mathrm{H}), 7.49(\mathrm{~d}, J=8.0 \mathrm{~Hz}, 2 \mathrm{H}$, Ar-2,6H), $7.28(\mathrm{~d}, J=2.0 \mathrm{~Hz}, 1 \mathrm{H}$, Pyrrole- $3 \mathrm{H}), 7.16(\mathrm{~d}, J=8.0 \mathrm{~Hz}, 2 \mathrm{H}, \mathrm{Ar}-3,5 \mathrm{H}), 5.63\left(\mathrm{~s}, 2 \mathrm{H}, \mathrm{NCH}_{2} \mathrm{O}\right)$, $3.76\left(\mathrm{~s}, 3 \mathrm{H}, \mathrm{CH}_{3}\right), 3.50\left(\mathrm{t}, J=8.0 \mathrm{~Hz}, 2 \mathrm{H}, \mathrm{OCH}_{2} \mathrm{CH}_{2} \mathrm{Si}\right), 2.29\left(\mathrm{~s}, 3 \mathrm{H}, \mathrm{CH}_{3}\right), 0.81(\mathrm{t}, J=8.0 \mathrm{~Hz}, 2 \mathrm{H}$, $\left.\mathrm{OCH}_{2} \mathrm{CH}_{2} \mathrm{Si}\right),-0.07$ (s, 9H, $\left.\mathrm{Si}\left(\mathrm{CH}_{3}\right)_{3}\right) .{ }^{13} \mathrm{C}-\mathrm{NMR}\left(100 \mathrm{MHz}, \mathrm{DMSO}-d_{6}\right) \delta: 161.0,135.7,131.4,129.8$, 127.1, 125.1, 123.9, 122.6, 116.0, 76.8, 65.5, 51.6, 21.1, 17.6, -1.0. HRMS (ESI): $m / z[M+\mathrm{H}]^{+}$calcd for $\mathrm{C}_{19} \mathrm{H}_{28} \mathrm{NO}_{3} \mathrm{Si}$ : 346.1833; found: 346.1839 .

Methyl 4-(o-tolyl)-1-((2-(trimethylsilyl)ethoxy)methyl)-1H-pyrrole-2-carboxylate (3m). Colorless oil, 52\% yield. ${ }^{1} \mathrm{H}-\mathrm{NMR}\left(400 \mathrm{MHz}, \mathrm{DMSO}-d_{6}\right) \delta: 7.52(\mathrm{~d}, J=2.0 \mathrm{~Hz}, 1 \mathrm{H}$, Pyrrole-5H), $7.32(\mathrm{dd}, J=7.6,2.0 \mathrm{~Hz}$, 1H, Ar-6H), 7.25-7.16 (m, 3H, Ar-3,4,5H), 7.09 (d, $J=2.0 \mathrm{~Hz}, 1 \mathrm{H}$, Pyrrole-3H), $5.66\left(\mathrm{~s}, 2 \mathrm{H}, \mathrm{NCH}_{2} \mathrm{O}\right), 3.76$ $\left(\mathrm{s}, 3 \mathrm{H}, \mathrm{CH}_{3}\right), 3.51\left(\mathrm{t}, J=8.0 \mathrm{~Hz}, 2 \mathrm{H}, \mathrm{OCH}_{2} \mathrm{CH}_{2} \mathrm{Si}\right), 2.37\left(\mathrm{~s}, 3 \mathrm{H}, \mathrm{CH}_{3}\right), 0.81\left(\mathrm{t}, J=8.0 \mathrm{~Hz}, 2 \mathrm{H}, \mathrm{OCH}_{2} \mathrm{CH}_{2} \mathrm{Si}\right)$, $-0.07\left(\mathrm{~s}, 9 \mathrm{H}, \mathrm{Si}\left(\mathrm{CH}_{3}\right)_{3}\right) .{ }^{13} \mathrm{C}-\mathrm{NMR}\left(100 \mathrm{MHz}, \mathrm{DMSO}-d_{6}\right) \delta: 161.0,135.1,134.2,131.1,129.5,129.2$, 127.0, 126.5, 123.4, 121.8, 119.1, 76.9, 65.5, 51.6, 21.5, 17.6, -1.0. HRMS (ESI): $m / z[\mathrm{M}+\mathrm{H}]^{+}$calcd for $\mathrm{C}_{19} \mathrm{H}_{28} \mathrm{NO}_{3} \mathrm{Si}$ : 346.1833; found: 346.1843 .

Methyl 4-(naphthalen-2-yl)-1-((2-(trimethylsilyl)ethoxy)methyl)-1H-pyrrole-2-carboxylate (3n). Colorless oil, 76\% yield. ${ }^{1} \mathrm{H}-\mathrm{NMR}\left(400 \mathrm{MHz}\right.$, DMSO- $\left.d_{6}\right) \delta: 8.16(\mathrm{dd}, J=7.2,2.4 \mathrm{~Hz}, 1 \mathrm{H}, \mathrm{Ar}-5 \mathrm{H}), 7.98-7.95$ $(\mathrm{m}, 1 \mathrm{H}, \operatorname{Ar}-4 \mathrm{H}), 7.87(\mathrm{~d}, J=8.0 \mathrm{~Hz}, 1 \mathrm{H}, \mathrm{Ar}-8 \mathrm{H}), 7.65(\mathrm{~d}, J=2.0 \mathrm{~Hz}, 1 \mathrm{H}$, Pyrrole-5H), 7.54-7.46 (m, $4 \mathrm{H}, \mathrm{Ar}-1,3,6,7 \mathrm{H}), 7.18\left(\mathrm{~d}, J=2.0 \mathrm{~Hz}, 1 \mathrm{H}\right.$, Pyrrole-3H), $5.73\left(\mathrm{~s}, 2 \mathrm{H}, \mathrm{NCH}_{2} \mathrm{O}\right), 3.79\left(\mathrm{~s}, 3 \mathrm{H}, \mathrm{CH}_{3}\right), 3.57(\mathrm{t}$, $\left.J=7.6 \mathrm{~Hz}, 2 \mathrm{H}, \mathrm{OCH}_{2} \mathrm{CH}_{2} \mathrm{Si}\right), 0.84\left(\mathrm{t}, J=7.6 \mathrm{~Hz}, 2 \mathrm{H}, \mathrm{OCH}_{2} \mathrm{CH}_{2} \mathrm{Si}\right),-0.05\left(\mathrm{~s}, 9 \mathrm{H}, \mathrm{Si}\left(\mathrm{CH}_{3}\right)_{3}\right) .{ }^{13} \mathrm{C}-\mathrm{NMR}$ $\left(100 \mathrm{MHz}\right.$, DMSO- $\left.d_{6}\right) \delta: 161.0,134.1,132.8,131.3,129.9,128.9,127.5,127.0,126.7,126.3,126.2,125.5$, 122.4, 122.2, 119.7, 77.0, 65.6, 51.7, 17.6, -1.0. HRMS (ESI): $\mathrm{m} / z[\mathrm{M}+\mathrm{H}]^{+}$calcd for $\mathrm{C}_{22} \mathrm{H}_{28} \mathrm{NO}_{3} \mathrm{Si}$ : 382.1833; found: 382.1839 .

Methyl 4-(quinolin-3-yl)-1-((2-(trimethylsilyl)ethoxy)methyl)-1H-pyrrole-2-carboxylate (3o). Colorless solid, $66 \%$ yield. M.p. $52-54{ }^{\circ} \mathrm{C} .{ }^{1} \mathrm{H}-\mathrm{NMR}\left(400 \mathrm{MHz}, \mathrm{DMSO}-d_{6}\right) \delta: 9.21(\mathrm{~d}, J=2.0 \mathrm{~Hz}, 1 \mathrm{H}, \mathrm{Ar}-2 \mathrm{H}), 8.53$ $(\mathrm{d}, J=2.0 \mathrm{~Hz}, 1 \mathrm{H}, \mathrm{Ar}-4 \mathrm{H}), 8.06(\mathrm{~d}, J=2.0 \mathrm{~Hz}, 1 \mathrm{H}$, Pyrrole- $5 \mathrm{H}), 7.94(\mathrm{~d}, J=8.4 \mathrm{~Hz}, 1 \mathrm{H}, \mathrm{Ar}-8 \mathrm{H}), 7.89$ $(\mathrm{d}, J=7.2 \mathrm{~Hz}, 1 \mathrm{H}, \mathrm{Ar}-5 \mathrm{H}), 7.65$ (td, $J=7.2,1.6 \mathrm{~Hz}, 1 \mathrm{H}, \mathrm{Ar}-6 \mathrm{H}), 7.57-7.53(\mathrm{~m}, 2 \mathrm{H}$, Ar-7H, Pyrrole- $3 \mathrm{H})$, $5.65\left(\mathrm{~s}, 2 \mathrm{H}, \mathrm{NCH}_{2} \mathrm{O}\right), 3.77\left(\mathrm{~s}, 3 \mathrm{H}, \mathrm{CH}_{3}\right), 3.51\left(\mathrm{t}, J=8.0 \mathrm{~Hz}, 2 \mathrm{H}, \mathrm{OCH}_{2} \mathrm{CH}_{2} \mathrm{Si}\right), 0.80(\mathrm{t}, J=8.0 \mathrm{~Hz}, 2 \mathrm{H}$, $\left.\mathrm{OCH}_{2} \mathrm{CH}_{2} \mathrm{Si}\right),-0.1$ (s, 9H, Si $\left.\left(\mathrm{CH}_{3}\right)_{3}\right) .{ }^{13} \mathrm{C}-\mathrm{NMR}\left(100 \mathrm{MHz}, \mathrm{DMSO}-d_{6}\right) \delta: 160.9,149.0,146.7,130.1,129.2$, 
129.1, 128.4, 128.3, 128.2, 127.5, 127.4, 123.2, 120.8, 116.5, 77.1, 65.7, 51.8, 17.6, -1.0. HRMS (ESI): $\mathrm{m} / \mathrm{z}$ [M $+\mathrm{H}]^{+}$calcd for $\mathrm{C}_{21} \mathrm{H}_{27} \mathrm{~N}_{2} \mathrm{O}_{3}$ Si: 383.1786 ; found: 383.1783 .

Methyl 4-(pyridin-3-yl)-1-((2-(trimethylsilyl)ethoxy)methyl)-1H-pyrrole-2-carboxylate (3p). Colorless oil, $41 \%$ yield. ${ }^{1} \mathrm{H}-\mathrm{NMR}\left(500 \mathrm{MHz}\right.$, DMSO- $\left.d_{6}\right) \delta: 8.88(\mathrm{~s}, 1 \mathrm{H}, \mathrm{Py}-2 \mathrm{H}), 8.40(\mathrm{~d}, J=3.5 \mathrm{~Hz}, 1 \mathrm{H}, \mathrm{Py}-6 \mathrm{H}), 8.01$ $(\mathrm{d}, J=7.5 \mathrm{~Hz}, 1 \mathrm{H}, \mathrm{Py}-4 \mathrm{H}), 7.93$ (s, 1H, Pyrrole-5H), $7.43(\mathrm{~s}, 1 \mathrm{H}$, Pyrrole-3H), 7.36 (dd, J = 7.5, $5.0 \mathrm{~Hz}$, $1 \mathrm{H}, \mathrm{Py}-5 \mathrm{H}), 5.65\left(\mathrm{~s}, 2 \mathrm{H}, \mathrm{NCH}_{2} \mathrm{O}\right), 3.78\left(\mathrm{~s}, 3 \mathrm{H}, \mathrm{CH}_{3}\right), 3.51\left(\mathrm{t}, J=8.0 \mathrm{~Hz}, 2 \mathrm{H}, \mathrm{OCH}_{2} \mathrm{CH}_{2} \mathrm{Si}\right), 0.82(\mathrm{t}, J=8.0$ $\left.\mathrm{Hz}, 2 \mathrm{H}, \mathrm{OCH}_{2} \mathrm{CH}_{2} \mathrm{Si}\right),-0.07\left(\mathrm{~s}, 9 \mathrm{H}, \mathrm{Si}\left(\mathrm{CH}_{3}\right)_{3}\right) .{ }^{13} \mathrm{C}-\mathrm{NMR}\left(100 \mathrm{MHz}, \mathrm{DMSO}-d_{6}\right) \delta: 160.9,147.6,146.5$, 132.3, 130.0, 127.9, 124.2, 123.1, 120.6, 116.2, 77.0, 65.7, 51.7, 17.5, -1.0. HRMS (ESI): $m / z$ [M + H] calcd for $\mathrm{C}_{17} \mathrm{H}_{25} \mathrm{~N}_{2} \mathrm{O}_{3} \mathrm{Si}$ : 333.1629; found: 333.1622 .

Methyl 4-(furan-2-yl)-1-((2-(trimethylsilyl)ethoxy)methyl)-1H-pyrrole-2-carboxylate (3q). Colorless oil, 45\% yield. ${ }^{1} \mathrm{H}-\mathrm{NMR}\left(500 \mathrm{MHz}, \mathrm{DMSO}-d_{6}\right) \delta: 7.61(\mathrm{~s}, 1 \mathrm{H}$, Furan-5H), $7.58(\mathrm{~s}, 1 \mathrm{H}$, Pyrrole-5H), $7.16(\mathrm{~s}, 1 \mathrm{H}$, Furan- $4 \mathrm{H}), 6.57\left(\mathrm{~d}, J=2.5 \mathrm{~Hz}, 1 \mathrm{H}\right.$, Furan-3H), $6.49(\mathrm{~s}, 1 \mathrm{H}$, Pyrrole- $3 \mathrm{H}), 5.64\left(\mathrm{~s}, 2 \mathrm{H}, \mathrm{NCH}_{2} \mathrm{O}\right), 3.76(\mathrm{~s}, 3 \mathrm{H}$, $\left.\mathrm{CH}_{3}\right), 3.49\left(\mathrm{t}, J=8.0 \mathrm{~Hz}, 2 \mathrm{H}, \mathrm{OCH}_{2} \mathrm{CH}_{2} \mathrm{Si}\right), 0.81\left(\mathrm{t}, J=8.0 \mathrm{~Hz}, 2 \mathrm{H}, \mathrm{OCH}_{2} \mathrm{CH}_{2} \mathrm{Si}\right),-0.08\left(\mathrm{~s}, 9 \mathrm{H}, \mathrm{Si}\left(\mathrm{CH}_{3}\right)_{3}\right)$. ${ }^{13} \mathrm{C}-\mathrm{NMR}\left(100 \mathrm{MHz}\right.$, DMSO- $\left.d_{6}\right) \delta: 160.8,149.6,141.5,126.1,122.6,115.6,115.1,111.9,104.1,76.9,65.6$, 51.7, 17.5, -1.0. HRMS (ESI): $m / z$ [M + H] calcd for $\mathrm{C}_{16} \mathrm{H}_{24} \mathrm{NO}_{4} \mathrm{Si}$ : 322.1469; found: 322.1483 .

Methyl 5-phenyl-1-((2-(trimethylsilyl)ethoxy)methyl)-1H-pyrrole-2-carboxylate (3r). Colorless oil, 95\% yield. ${ }^{1} \mathrm{H}-\mathrm{NMR}\left(500 \mathrm{MHz}, \mathrm{DMSO}-d_{6}\right) \delta: 7.55(\mathrm{~d}, J=7.5 \mathrm{~Hz}, 2 \mathrm{H}, \mathrm{Ar}-2,6 \mathrm{H}), 7.49-7.44(\mathrm{~m}, 3 \mathrm{H}, \mathrm{Ar}-3,4,5 \mathrm{H}), 7.03$ $(\mathrm{d}, J=3.5 \mathrm{~Hz}, 1 \mathrm{H}$, Pyrrole- $3 \mathrm{H}), 6.33(\mathrm{~d}, J=4.0 \mathrm{~Hz}, 1 \mathrm{H}$, Pyrrole- $4 \mathrm{H}), 5.62\left(\mathrm{~s}, 2 \mathrm{H}, \mathrm{NCH}_{2} \mathrm{O}\right), 3.77(\mathrm{~s}, 3 \mathrm{H}$, $\left.\mathrm{CH}_{3}\right), 3.36\left(\mathrm{t}, J=8.0 \mathrm{~Hz}, 2 \mathrm{H}, \mathrm{OCH}_{2} \mathrm{CH}_{2} \mathrm{Si}\right), 0.73\left(\mathrm{t}, J=8.0 \mathrm{~Hz}, 2 \mathrm{H}, \mathrm{OCH}_{2} \mathrm{CH}_{2} \mathrm{Si}\right),-0.10\left(\mathrm{~s}, 9 \mathrm{H}, \mathrm{Si}\left(\mathrm{CH}_{3}\right)_{3}\right)$. ${ }^{13} \mathrm{C}-\mathrm{NMR}\left(100 \mathrm{MHz}\right.$, DMSO- $\left.d_{6}\right) \delta: 161.2,142.5,131.8,129.5,129.2,128.8,123.3,119.3,110.3,73.4,65.3$, 51.6, 17.9, -1.0. HRMS (ESI): $m / z[\mathrm{M}+\mathrm{H}]^{+}$calcd for $\mathrm{C}_{18} \mathrm{H}_{26} \mathrm{NO}_{3} \mathrm{Si}$ : 332.1677; found: 332.1661.

Methyl 3-phenyl-1-((2-(trimethylsilyl)ethoxy)methyl)-1H-pyrrole-2-carboxylate (3s). Colorless solid, 99\% yield. M.p. $87-88^{\circ} \mathrm{C} .{ }^{1} \mathrm{H}-\mathrm{NMR}\left(500 \mathrm{MHz}\right.$, DMSO- $\left.d_{6}\right) \delta$ : 7.35-7.30 (m, 6H, Ar-2,3,4,5,6H, Pyrrole-5H), $6.24(\mathrm{~d}, J=2.0 \mathrm{~Hz}, 1 \mathrm{H}$, Pyrrole- $4 \mathrm{H}), 5.60\left(\mathrm{~s}, 2 \mathrm{H}, \mathrm{NCH}_{2} \mathrm{O}\right), 3.58\left(\mathrm{~s}, 3 \mathrm{H}, \mathrm{CH}_{3}\right), 3.46(\mathrm{t}, J=7.5 \mathrm{~Hz}, 2 \mathrm{H}$, $\left.\mathrm{OCH}_{2} \mathrm{CH}_{2} \mathrm{Si}\right), 0.81\left(\mathrm{t}, J=7.5 \mathrm{~Hz}, 2 \mathrm{H}, \mathrm{OCH}_{2} \mathrm{CH}_{2} \mathrm{Si}\right),-0.06\left(\mathrm{~s}, 9 \mathrm{H}, \mathrm{Si}\left(\mathrm{CH}_{3}\right)_{3}\right) .{ }^{13} \mathrm{C}-\mathrm{NMR}(100 \mathrm{MHz}$, DMSO- $d_{6}$ ) $\delta: 161.8,136.3,134.2,129.4,129.2,128.2,127.1,118.1,110.6,77.6,65.5,51.3,17.6,-1.0$. HRMS (ESI): $m / z[\mathrm{M}+\mathrm{H}]^{+}$calcd for $\mathrm{C}_{18} \mathrm{H}_{26} \mathrm{NO}_{3} \mathrm{Si}$ : 332.1677; found: 332.1663 .

\subsection{Procedure for the Preparation of Methyl 4-phenyl-1H-pyrrole-2-carboxylate (4a)}

A mixture of 3a (500 mg, $1.5 \mathrm{mmol})$ and TBAF ( $3 \mathrm{~mL}, 1 \mathrm{~mol} / \mathrm{L}$ THF) was stirred at $80^{\circ} \mathrm{C}$ for $2 \mathrm{~h}$ and cooled to rt. Then, the reaction mixture was diluted with EtOAc and $\mathrm{H}_{2} \mathrm{O}$. The aqueous phase was extracted with EtOAc, and the combined organic layer was washed with brine, dried with $\mathrm{Na}_{2} \mathrm{SO}_{4}$, and concentrated. Purification by flash chromatography (EtOAc in $\mathrm{PE}=14 \%$ ) gave $4 \mathbf{a}(251 \mathrm{mg}, 83 \%)$ as a colorless solid. M.p. $198-200{ }^{\circ} \mathrm{C} .{ }^{1} \mathrm{H}-\mathrm{NMR}\left(500 \mathrm{MHz}, \mathrm{DMSO}-d_{6}\right) \delta: 12.07(\mathrm{~s}, 1 \mathrm{H}, \mathrm{NH}), 7.62(\mathrm{~d}, J=$ $7.0 \mathrm{~Hz}, 2 \mathrm{H}, \mathrm{Ar}-2,6 \mathrm{H}), 7.51(\mathrm{~s}, 1 \mathrm{H}$, Pyrrole- $5 \mathrm{H}), 7.32(\mathrm{t}, J=7.0 \mathrm{~Hz}, 2 \mathrm{H}, \mathrm{Ar}-3,5 \mathrm{H}), 7.19-7.16(\mathrm{~m}, 2 \mathrm{H}, \mathrm{Ar}-4 \mathrm{H}$, Pyrrole-3H), $3.79\left(\mathrm{~s}, 3 \mathrm{H}, \mathrm{CH}_{3}\right) .{ }^{1} \mathrm{H}-\mathrm{NMR}$ data was in agreement with the literature [28]. ${ }^{13} \mathrm{C}-\mathrm{NMR}(100$ MHz, DMSO- $d_{6}$ ) $\delta: 161.3,135.0,129.1,126.2,125.6,125.2,123.2,121.7,112.4,51.6$. HRMS (ESI): $m / z$ [M + $\mathrm{H}]^{+}$calcd for $\mathrm{C}_{12} \mathrm{H}_{12} \mathrm{NO}_{2}$ : 202.0863; found: 202.0856 .

3.5. Procedure for the Preparation of 4-Phenyl-1-((2-(trimethylsilyl)ethoxy)methyl)-1H-pyrrole-2-carboxylic acid (4b)

A mixture of $3 \mathrm{a}(250 \mathrm{mg}, 0.75 \mathrm{mmol})$ and $\mathrm{NaOH}(60 \mathrm{mg}, 1.5 \mathrm{mmol})$ in ethanol $(6 \mathrm{~mL})$ and $\mathrm{H}_{2} \mathrm{O}$ $(4 \mathrm{~mL})$ was stirred at $100{ }^{\circ} \mathrm{C}$ for $6 \mathrm{~h}$. Then, the reaction mixture was concentrated to remove ethanol, and the residue was adjusted to $\mathrm{pH} 7$ with $\mathrm{HCl}(2 \mathrm{M})$. The aqueous phase was extracted with EtOAc, and the combined organic layer was washed with brine, dried with $\mathrm{Na}_{2} \mathrm{SO}_{4}$, and concentrated to give $4 \mathbf{b}(200 \mathrm{mg}, 84 \%)$ as a colorless solid. M.p. 83-85 ${ }^{\circ} \mathrm{C} .{ }^{1} \mathrm{H}-\mathrm{NMR}\left(400 \mathrm{MHz}, \mathrm{DMSO}-d_{6}\right) \delta: 12.44(\mathrm{~s}, 1 \mathrm{H}$, $\mathrm{COOH}), 7.72(\mathrm{~d}, J=1.6 \mathrm{~Hz}, 1 \mathrm{H}$, Pyrrole- $5 \mathrm{H}), 7.59(\mathrm{~d}, J=7.2 \mathrm{~Hz}, 2 \mathrm{H}, \mathrm{Ar}-2,6 \mathrm{H}), 7.34(\mathrm{t}, J=7.6 \mathrm{~Hz}, 2 \mathrm{H}$, Ar-3,5H), $7.26(\mathrm{~d}, J=2.0 \mathrm{~Hz}, 1 \mathrm{H}$, Pyrrole- $3 \mathrm{H}), 7.18(\mathrm{t}, J=7.6 \mathrm{~Hz}, 1 \mathrm{H}, \mathrm{Ar}-4 \mathrm{H}), 5.66\left(\mathrm{~s}, 2 \mathrm{H}, \mathrm{NCH}_{2} \mathrm{O}\right), 3.51$ 
$\left(\mathrm{t}, J=8.0 \mathrm{~Hz}, 2 \mathrm{H}, \mathrm{OCH}_{2} \mathrm{CH}_{2} \mathrm{Si}\right), 0.82\left(\mathrm{t}, J=8.0 \mathrm{~Hz}, 2 \mathrm{H}, \mathrm{OCH}_{2} \mathrm{CH}_{2} \mathrm{Si}\right),-0.07\left(\mathrm{~s}, 9 \mathrm{H}, \mathrm{Si}\left(\mathrm{CH}_{3}\right)_{3}\right) .{ }^{13} \mathrm{C}-\mathrm{NMR}$ $\left(100 \mathrm{MHz}, \mathrm{DMSO}-d_{6}\right) \delta: 162.2,134.4,129.2,126.8,126.5,125.1,123.9,123.6,115.9,76.7,65.5,17.6,-0.9$. HRMS (ESI): $m / z[\mathrm{M}+\mathrm{H}]^{+}$calcd for $\mathrm{C}_{17} \mathrm{H}_{24} \mathrm{NO}_{3} \mathrm{Si}: 318.1520$; found: 318.1489 .

\subsection{Procedure for the Preparation of (4-Phenyl-1-((2-(trimethylsilyl)ethoxy)methyl)-1H-pyrrol-2-yl)methanol (4c)}

First, $\mathrm{LiAlH}_{4}(35 \mathrm{mg}, 0.94 \mathrm{mmol})$ was added to a solution of $3 \mathrm{a}(155 \mathrm{mg}, 0.47 \mathrm{mmol})$ in anhydrous THF (6 mL) at rt. After stirring overnight, the reaction mixture was quenched with $15 \% \mathrm{NaOH}(0.11$ $\mathrm{mL}$ ) and extracted with EtOAc/ $\mathrm{H}_{2} \mathrm{O}$, and the organic layer was washed with brine, dried with $\mathrm{Na}_{2} \mathrm{SO}_{4}$, and concentrated. Purification by flash chromatography (EtOAc in PE $=14 \%$ ) gave $4 \mathrm{c}(127 \mathrm{mg}, 89 \%)$ as a yellow oil. ${ }^{1} \mathrm{H}-\mathrm{NMR}\left(500 \mathrm{MHz}, \mathrm{DMSO}-d_{6}\right) \delta: 7.49(\mathrm{~d}, J=7.5 \mathrm{~Hz}, 2 \mathrm{H}, \mathrm{Ar}-2,6 \mathrm{H}), 7.31-7.28(\mathrm{~m}, 3 \mathrm{H}$, Ar-3,5H, Pyrrole-5H), 7.11 (t, J = 7.5 Hz, 1H, Ar-4H), 6.41 (s, 1H, Pyrrole-3H), 5.29 (s, 2H, NCH $2 \mathrm{O}), 4.98$ (brs, $1 \mathrm{H}, \mathrm{OH}), 4.47\left(\mathrm{~d}, \mathrm{~J}=4.0 \mathrm{~Hz}, 2 \mathrm{H}, \mathrm{CH}_{2} \mathrm{OH}\right), 3.50\left(\mathrm{t}, J=8.0 \mathrm{~Hz}, 2 \mathrm{H}, \mathrm{OCH}_{2} \mathrm{CH}_{2} \mathrm{Si}\right), 0.84(\mathrm{t}, J=8.0 \mathrm{~Hz}$, $\left.2 \mathrm{H}, \mathrm{OCH}_{2} \mathrm{CH}_{2} \mathrm{Si}\right),-0.04\left(\mathrm{~s}, 9 \mathrm{H}, \mathrm{Si}\left(\mathrm{CH}_{3}\right)_{3}\right) .{ }^{13} \mathrm{C}-\mathrm{NMR}\left(100 \mathrm{MHz}, \mathrm{DMSO}-d_{6}\right) \delta: 135.9,134.7,129.1,125.5$, 124.7, 122.6, 120.0, 107.1, 76.0, 65.2, 55.3, 17.6, -0.9. HRMS (ESI): $m / z$ [M + H] $]^{+}$calcd for $\mathrm{C}_{17} \mathrm{H}_{26} \mathrm{NO}_{2} \mathrm{Si}$ : 304.1727; found: 304.1708 .

\section{Conclusions}

In summary, we have developed an efficient Suzuki-Miyaura coupling reaction of SEM-protected bromopyrroles and arylboronic acids under mild conditions leading to aryl-substituted pyrroles. This methodology is a practical and straightforward way to synthesize various pyrrole building blocks for delivering novel compounds with broad functional group compatibility. The protocol displays some advantages compared to the reported methods, such as commercially available materials, no debrominated by-products being formed, and the SEM-protecting group being more tolerant than the BOC-protecting group under the reaction conditions. Around the privileged SEM-protected aryl-substituted pyrroles, we demonstrate the versatility of the pyrrole ring framework in synthetic transformations for constructing new structures. This protocol will offer the opportunity to explore other metal-catalyzed cross-coupling reactions using SEM-protected pyrroles and generate new bioactive compounds containing polyfunctionalized pyrroles.

Supplementary Materials: The following are available online: ${ }^{1} \mathrm{H}-\mathrm{NMR}$ and HRMS of compounds $2 \mathbf{a}-\mathbf{c},{ }^{1} \mathrm{H}-\mathrm{NMR}$, ${ }^{13} \mathrm{C}-\mathrm{NMR}$ and HRMS of compounds $3 \mathbf{a}-\mathbf{s}$ and $4 \mathbf{a}-\mathbf{c}$.

Author Contributions: K.C. and M.G. performed the experiments. H.Z. supervised the study. D.Z. and H.H. conceived and designed the study. D.Z., H.Y., and H.H. interpreted the results, and wrote the paper.

Funding: This research was funded by the CAMS Innovation Fund for Medical Sciences (CIFMS) (Grant No. 2016-I2M-1-013) and National Science \& Technology Major Project of China (Grant No. 2015ZX09102007-012).

Conflicts of Interest: The authors declare no conflict of interest.

\section{References}

1. Ma, Z.N.; Ma, Z.C.; Zhang, D.W. Synthesis of multi-substituted pyrrole derivatives through [3+2] cycloaddition with tosylmethyl isocyanides (TosMICs) and electron-deficient compounds. Molecules 2018, 23, 2666. [CrossRef] [PubMed]

2. Hao, F.; Reddy, A.R.; Zhou, C.Y.; Che, C.M. Cobalt(II) porphyrin catalyzed cascade reaction of pyrrolyl ketones for construction of polysubstituted pyrrolizidines and pyrrolizines. Adv. Synth. Catal. 2018, 360, 1433-1438. [CrossRef]

3. Guillon, J.; Le Borgne, M.; Rimbault, C.; Moreau, S.; Savrimoutou, S.; Pinaud, N.; Baratin, S.; Marchivie, M.; Roche, S.; Bollacke, A.; et al. Synthesis and biological evaluation of novel substituted pyrrolo[1,2-a]quinoxaline derivatives as inhibitors of the human protein kinase CK2. Eur. J. Med. Chem. 2013, 65, 205-222. [CrossRef] 
4. Desplat, V.; Moreau, S.; Gay, A.; Fabre, S.B.; Thiolat, D.; Massip, S.; Macky, G.; Godde, F.; Mossalayi, D.; Jarry, C.; et al. Synthesis and evaluation of the antiproliferative activity of novel pyrrolo[1,2-a]quinoxaline derivatives, potential inhibitors of Akt kinase. Part II. J. Enzyme. Inhib. Med. Chem. 2010, 25, $204-215$. [CrossRef] [PubMed]

5. Rahman, K.M.; Jackson, P.J.; James, C.H.; Basu, B.P.; Hartley, J.A.; de la Fuente, M.; Schatzlein, A.; Robson, M.; Pedley, R.B.; Pepper, C.; et al. GC-targeted C8-linked pyrrolobenzodiazepine-biaryl conjugates with femtomolar in vitro cytotoxicity and in vivo antitumor activity in mouse models. J. Med. Chem. 2013, 56, 2911-2935. [CrossRef]

6. Burnham, B.S.; Berkowitz, J.D.; Orr, Z.; McCranor, B.; Cooper, M.; McCann, K.; Pillitteri, K.; Hallback, J.; Bergh, L.-M.; Gofman, L.; et al. Lipid-lowering effects of polymers derived from halophenyl pyrroles. Lett. Drug Des. Discov. 2011, 8, 544-549. [CrossRef]

7. Lopez-Perez, A.; Robles-Machin, R.; Adrio, J.; Carretero, J.C. Oligopyrrole Synthesis by 1,3-dipolar cycloaddition of azomethine ylides with bissulfonyl ethylenes. Angew. Chem. Int. Ed. 2007, 46, 9261-9264. [CrossRef]

8. Gao, M.; He, C.; Chen, H.; Bai, R.; Cheng, B.; Lei, A. Synthesis of pyrroles by click reaction: Silver-catalyzed cycloaddition of terminal alkynes with isocyanides. Angew. Chem. Int. Ed. 2013, 52, 6958-6961. [CrossRef] [PubMed]

9. Farney, E.P.; Yoon, T.P. Visible-light sensitization of vinyl azides by transition-metal photocatalysis. Angew. Chem. Int. Ed. 2014, 53, 793-797. [CrossRef]

10. O'Brien, A.G.; Levesque, F.; Seeberger, P.H. Continuous flow thermolysis of azidoacrylates for the synthesis of heterocycles and pharmaceutical intermediates. Chem. Commun. 2011, 47, 2688-2690. [CrossRef]

11. Tiwari, D.K.; Pogula, J.; Sridhar, B.; Tiwari, D.K.; Likhar, P.R. Nano-copper catalysed highly regioselective synthesis of 2,4-disubstituted pyrroles from terminal alkynes and isocyanides. Chem. Commun. 2015, 51, 13646-13649. [CrossRef]

12. Kudryavtsev, K.V.; Ivantcova, P.M.; Churakov, A.V.; Vasin, V.A. Phenyl $\alpha$-bromovinyl sulfone in cycloadditions with azomethine ylides: An unexpected facile aromatization of the cycloadducts into pyrroles. Tetrahedron Lett. 2012, 53, 4300-4303. [CrossRef]

13. Pasko, C.M.; Dissanayake, A.A.; Billow, B.S.; Odom, A.L. One-pot synthesis of pyrroles using a titanium-catalyzed multicomponent coupling procedure. Tetrahedron 2016, 72, 1168-1176. [CrossRef]

14. Chemler, S.R.; Fuller, P.H. Heterocycle synthesis by copper facilitated addition of heteroatoms to alkenes, alkynes and arenes. Chem. Soc. Rev. 2007, 36, 1153-1160. [CrossRef] [PubMed]

15. Dowlut, M.; Mallik, D.; Organ, M.G. An efficient low-temperature Stille-Migita cross-coupling reaction for heteroaromatic compounds by Pd-PEPPSI-IPent. Chem. Eur. J. 2010, 16, 4279-4283. [CrossRef]

16. Castro, M.C.R.; Raposo, M.M.M. Synthesis of $\pi$-conjugated systems bearing thiophene and pyrrole heterocycles through palladium catalyzed cross-coupling reactions. Tetrahedron 2016, 72, 1881-1887. [CrossRef]

17. Rodríguez, N.; Goossen, L.J. Decarboxylative coupling reactions: A modern strategy for C-C-bond formation. Chem. Soc. Rev. 2011, 40, 5030-5048. [CrossRef] [PubMed]

18. Yuan, K.; Souleé, J.-F.; Doucet, H. Functionalization of C-H Bonds via Metal-Catalyzed Desulfitative Coupling: An Alternative Tool for Access to Aryl- or Alkyl-Substituted (Hetero)arenes. ACS Catal. 2015, 5, 978-991. [CrossRef]

19. Handy, S.T.; Bregman, H.; Lewis, J.; Zhang, X.; Zhang, Y. An unusual dehalogenation in the Suzuki coupling of 4-bromopyrrole-2-carboxylates. Tetrahedron Lett. 2003, 44, 427-430. [CrossRef]

20. Smith, J.A.; Ng, S.; White, J. The regioselective synthesis of aryl pyrroles. Org. Biomol. Chem. 2006, 4, 2477-2482. [CrossRef]

21. Handy, S.T.; Zhang, Y.; Bregman, H. A Modular synthesis of the lamellarins: Total synthesis of lamellarin G trimethyl ether. J. Org. Chem. 2004, 69, 2362-2366. [CrossRef]

22. Muchowski, J.M.; Solas, D.R. Protecting groups for the pyrrole and indole nitrogen atom. the [2-(trimet hy1silyl)ethoxy]methyl moiety. lithiation of 1-[[2-(trimethylsilyl)ethoxy]methyl]pyrrole. J. Org. Chem. 1984, 49, 203-205. [CrossRef]

23. Whitten, J.P.; Matthews, D.P.; McCarthy, J.R. [2-(Trimethylsilyl)ethoxy]methyl (SEM) as a novel and effective imidazole and fused aromatic imidazole protecting group. J. Org. Chem. 1986, 51, 1891-1894. [CrossRef] 
24. Katz, J.D.; Overman, L.E. Studies towards the total synthesis of palau'amine. Formation of 4,5-dihydropyrrole-2-carboxylate intermediates by alkene-enamide ring-closing metathesis. Tetrahedron 2004, 60, 9559-9568. [CrossRef]

25. Toure, B.B.; Lane, B.S.; Sames, D. Catalytic C-H arylation of SEM-protected azoles with palladium complexes of NHCs and phosphines. Org. Lett. 2006, 8, 1979-1982. [CrossRef]

26. Yamaguchi, J.; Seiple, I.B.; Young, I.S.; O'Malley, D.P.; Maue, M.; Baran, P.S. Synthesis of 1,9-dideoxy-pre-axinellamine. Angew. Chem. Int. Ed. 2008, 47, 3578-3580. [CrossRef]

27. Sadler, S.A.; Hones, A.C.; Roberts, B.; Blakemore, D.; Marder, T.B.; Steel, P.G. Multidirectional synthesis of substituted indazoles via iridium-catalyzed C-H borylation. J. Org. Chem. 2015, 80, 5308-5314. [CrossRef] [PubMed]

28. Mccomas, C.C.; Serrano-wu, M.H.; Vacca, J.P. Fused quadracyclic compounds, compositions and uses thereof. U.S. Patent 20170190713A1, 6 July 2017.

Sample Availability: Samples of the compounds 3a-s and $4 \mathbf{a}-\mathbf{c}$ are available from the authors.

(C) 2019 by the authors. Licensee MDPI, Basel, Switzerland. This article is an open access article distributed under the terms and conditions of the Creative Commons Attribution (CC BY) license (http://creativecommons.org/licenses/by/4.0/). 